\title{
Lacrimal Gland Adenocarcinoma with Apocrine Metaplasia: A Case Report
}

\author{
Manabu Nishida*, Takayuki Taruya, Takashi Kono, Takao Hamamoto, Takashi Ishino, Tsutomu Ueda \\ and Sachio Takeno
}

\begin{abstract}
Department of Otolaryngology, Head and Neck Surgery, Division of Clinical Medical Science, Programs for Applied Biomedicine, Graduate School of Biomedical Sciences, Hiroshima University, Hiroshima, Japan
\end{abstract}

\begin{abstract}
We present the case of lacrimal gland adenocarcinoma with apocrine metaplasia and a literature review for similar cases. A 65-year-old male with painless eyelid swelling was admitted to our hospital. A physical examination revealed tumor on the caudal side of the left orbital cavity, and a biopsy indicated apocrine adenocarcinoma. The patient underwent a total left orbital exenteration with eyelid resection and reconstruction using a deep inferior epigastric perforator skin flap. The pathological evaluation led to a definitive diagnosis of lacrimal gland adenocarcinoma with apocrine metaplasia. Lacrimal carcinomas are rare in the head and neck region, so there are few studies of lacrimal gland carcinoma with apocrine metaplasia. The rarity hinders extensive elucidation of the pathological characteristics and evidence-based treatment strategies.
\end{abstract}

Keywords

Apocrine, Metaplasia, Adenocarcinoma, Lacrimal gland, Epigastric skin flap

\section{Introduction}

Tumors arising from lacrimal glands, which are rare and account for $9 \%$ of all orbital tumors, can be classified into epithelial (40\%) and lymphoid (60\%) tumors [1]. Lacrimal gland adenocarcinoma is extremely rare, comprising $2.8 \%$ of all epithelial tumors of the orbital cavity [2]. Although adenocystic carcinoma is the most frequent orbital epithelial malignancy, lacrimal gland adenocarcinoma has a worse prognosis than adenocystic carcinoma [3]. We describe the case of a patient with lacrimal gland adenocarcinoma, originating from the inferior eyelid, who was successfully treated with surgery and postoperative radiochemotherapy.

\section{Case Report}

A 65-year-old male experiencing painless swelling in inferior eyelid was admitted to an ophthalmology clinic. His medical history included hypertension, hepatitis $B$ infection, and claustrophobia, and his family history was unremarkable. A physical examination did not reveal pain or altered ocular movements except for a slight deviation to the superolateral side in the left eye. Computed tomography (CT) imaging revealed a tumor in the left orbital cavity. The patient was admitted to our hospital for further examination and treatment.

Upon admission, a granulation-like tumor on the inside of the left inferior eyelid was observed, and pathological evaluation of the incisional biopsy revealed apocrine adenocarcinoma. The patient did not consent to inferior eyelid resection and sought a second opinion at another hospital. However, he returned to our hospital four months later, after exploring nonsurgical treatment approaches, and provided informed consent to undergo surgery.
A preoperative physical examination revealed progression of the left ocular deviation, extroversion restriction of the eyeball, and a solid mass that was elastic to the touch in the inferior eyelid. CT revealed the tumor had extended backward to the external rectus muscle (Figure 1a and Figure 1b). Magnetic resonance imaging revealed invasion of the external rectus muscle, lacrimal gland, and ocular globe (Figure 1c). The positron emission tomography-CT scan showed focal ${ }^{18} \mathrm{~F}$-fluorodeoxyglucose uptake in the primary tumor with no evidence of lymph node or distant metastases (Figure 1d). Based on these findings, the patient was diagnosed with apocrine adenocarcinoma, arising from the inferior eyelid, and underwent radical surgery with reconstruction.

Briefly, total orbital exenteration with eyelid resection and reconstruction, using deep inferior epigastric perforator (DIEP) skin flap, was performed under general anesthesia. A circular skin incision with a $1.5-2.0 \mathrm{~cm}$ margin from the edge of the orbital bone was made (Figure 2a and Figure 2b). The tumor and the optical contents,

*Corresponding author: Manabu Nishida, Department of Otolaryngology, Hiroshima University, Kasumi 1-2-3, Minami-ku, Hiroshima 734-8551, Japan, Tel: 81-82-257-5252, Fax: 81-82257-5254

Accepted: June 11, 2019

Published online: June 13, 2019

Citation: Nishida M, Taruya T, Kono T, et al. (2019) Lacrimal Gland Adenocarcinoma with Apocrine Metaplasia: A Case Report. J Head Neck Surg 2(1):24-28 

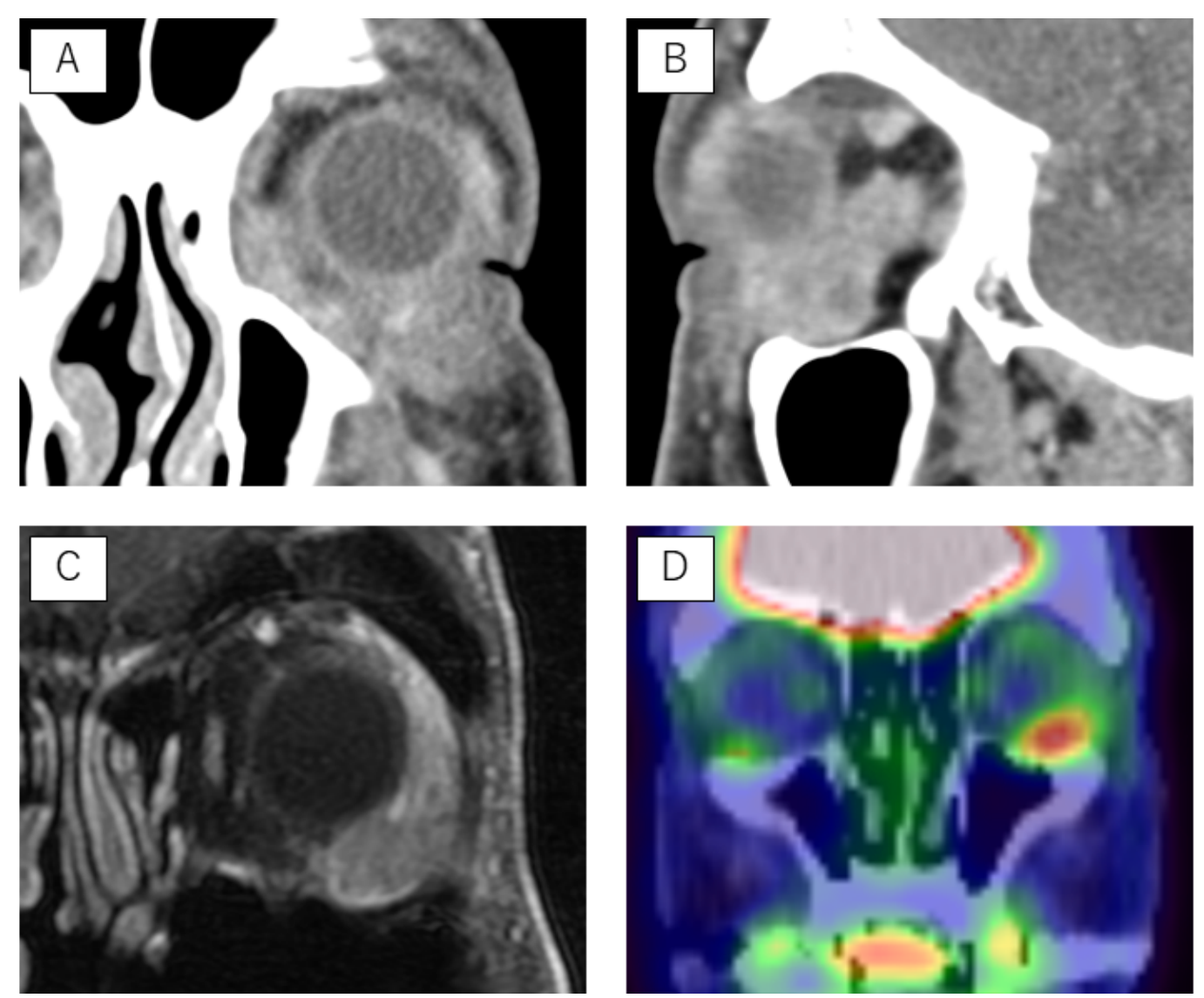

Figure 1: (a) Coronal contrast computed tomography (CT) scan; (b) Sagittal contrast CT scan; (c) Coronal gadolinium-enhanced T1weighted magnetic resonance image; (d) Coronal positron emission tomography-CT scan.
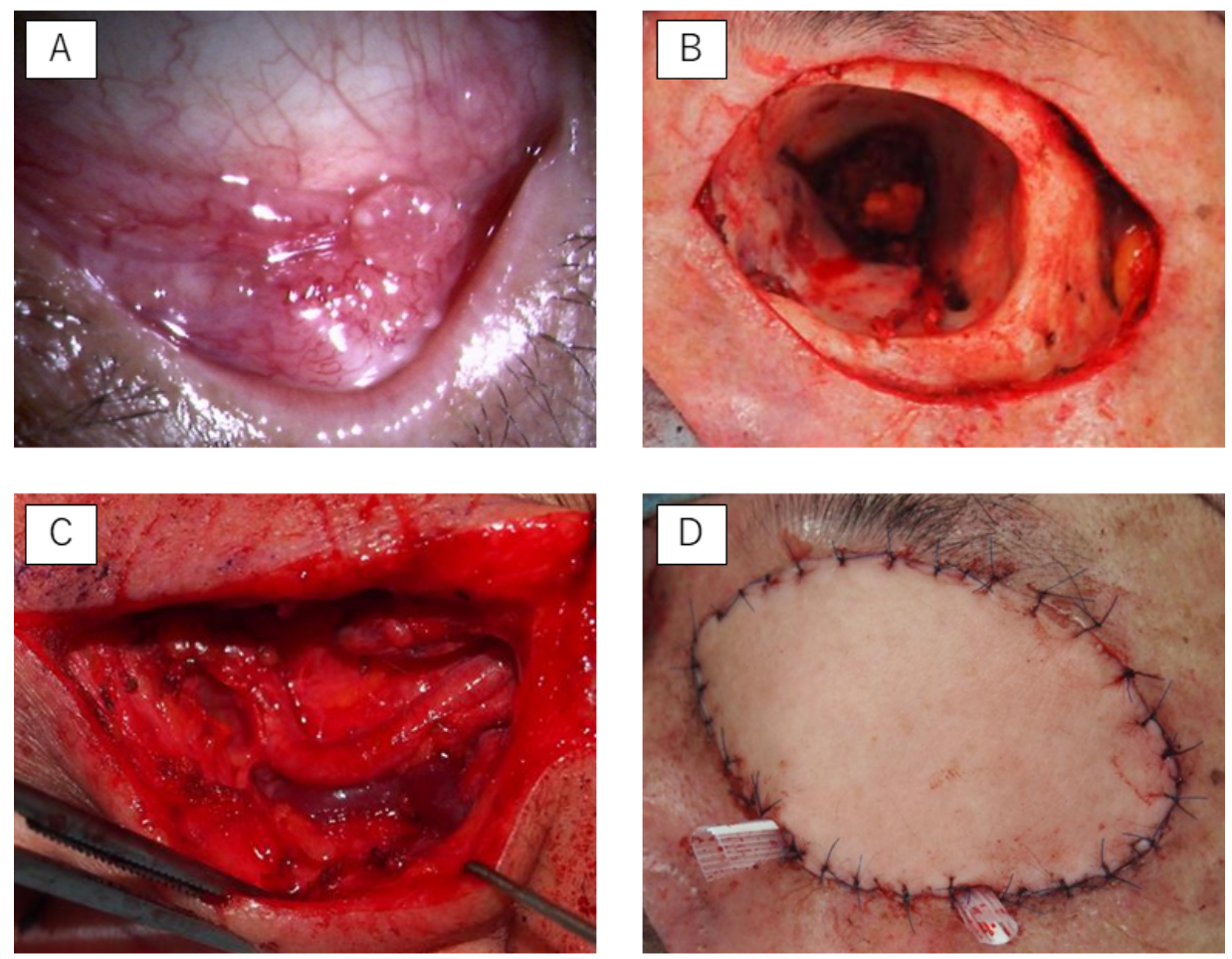

Figure 2: (a) Pre-operation; (b) Total orbital exenteration with eyelid resection; (c) Vascular anastomosis between the superficial temporal artery/vein and the inferior deep epigastric artery/vein; (d) Reconstruction with deep inferior epigastric perforator skin flap under general anesthesia. 
including the optical periosteum, were resected. The supraorbital and infraorbital nerves, anterior angular artery, anterior ethmoidal artery, all external ocular muscles, optic nerve, and ophthalmic artery were sacrificed. The DIEP skin flap was extracted from the left abdominal area, and vascular anastomoses were created between the superficial temporal artery/vein and the inferior deep epigastric artery/vein (Figure $2 \mathrm{c}$ and Figure $2 \mathrm{~d}$ ).

Postoperative pathological examination revealed that the tumor, $20 \times 15 \times 12 \mathrm{~mm}$ in dimensions, had invaded the lacrimal gland ocular globe, palpebral conjunctiva, external rectus muscle, and surrounding tissues. Hematoxylin/eosin staining showed polygonal tumor cells with multiple, irregular-shaped nuclei and acidophilic granular cytoplasm. Perineural, lymphatic, and vascular invasion were recognized, and the tumor margin at the edge of the external rectus muscle was not free. Although there were no tumor cells invading Moll's glands of the inferior eyelid (Figure 3), some parts of the tumor had structures resembling secretory glands (Figure 4a). Immunohistochemical analysis showed that the tumor was positive for cytokeratin, epithelial membrane antigen, and gross cystic disease fluid protein-15 (GCDFP-15) (Figure 4b) and negative for androgen receptor, carcinoembryonic antigen, $\alpha$-smooth muscle actin, and S-100. Pathological findings and immunohistochemical analysis led to the definitive diagnosis of lacrimal gland adenocarcinoma with apocrine metaplasia arising from the inferior eyelid (Figure 4c).

Since the surgical margin was not free, the patient received postoperative chemoradiotherapy (two cycles of $100 \mathrm{mg} / \mathrm{m}^{2}$ cisplatin plus intensity-modulated radiotherapy $60 \mathrm{~Gy}$ ). The patient showed no recurrence at three years after treatment and was alive at last follow-up.

\section{Discussion}

Lacrimal gland lesions represent $9 \%$ of all space-occupying orbital lesions [1] and predominantly include lymphomas and inflammatory lesions. Primary epithelial tumors represent less than $40 \%$ of all lacrimal gland lesions [1,2]. Adenocarcinoma is extremely rare, accounting for $2.8 \%$ [2], so staging and treatment strategies are not established. However, the prognosis of adenocarcinoma is considered to be worse than that of adenocystic carcinoma, which is the most common malignant lacrimal gland tumor [3].

Tumors of the lacrimal gland, located in the superolateral orbital cavity, deviate the ocular ball to the inferomedial side. In the current patient, we recognized the primary lesion was not in the lacrimal gland because the ocular deviation was superolateral, and the focal ${ }^{18} \mathrm{~F}$-fluorodeoxyglucose uptake was in the inferior eyelid. Moll's glands are the only apocrine glands in the orbital cavity; therefore, the preoperative diagnosis was apocrine adenocarcinoma of the Moll's glands of the inferior eyelid. However, the definitive diagnosis, based on pathological and immunohistological evaluation, was lacrimal gland adenocarcinoma with apocrine metaplasia.

Apocrine adenocarcinoma, apocrine carcinoma, and carcinoma with apocrine metaplasia have distinct implications. Apocrine adenocarcinoma is a malignant tumor of the apocrine gland. Apocrine carcinoma is a malignant tumor with apocrine metaplasia in more than $90 \%$ of the lesions, and carcinoma with apocrine metaplasia is a partially differentiated malignant tumor with apocrine gland-like configuration.

Kipkie and Paties suggested apocrine carcinomas should fulfill the following histopathological criteria for $[4,5]$ : tumor cells in an anatomical location where apocrine glands exist, periodic acidSchiff-positive and diastase-resistant material in cells or luminal spaces, decapitation secretion, and immunoreactivity for GCDFP-15. Requena, et al. also reported tumors with apocrine metaplasia were characterized by decapitation secretion, i.e., fluid secretion into the lumen of lobules via decapitation, and GCDFP-15 immunoreactivity [6].

In the current case, the tumor cells located mainly in the lac-

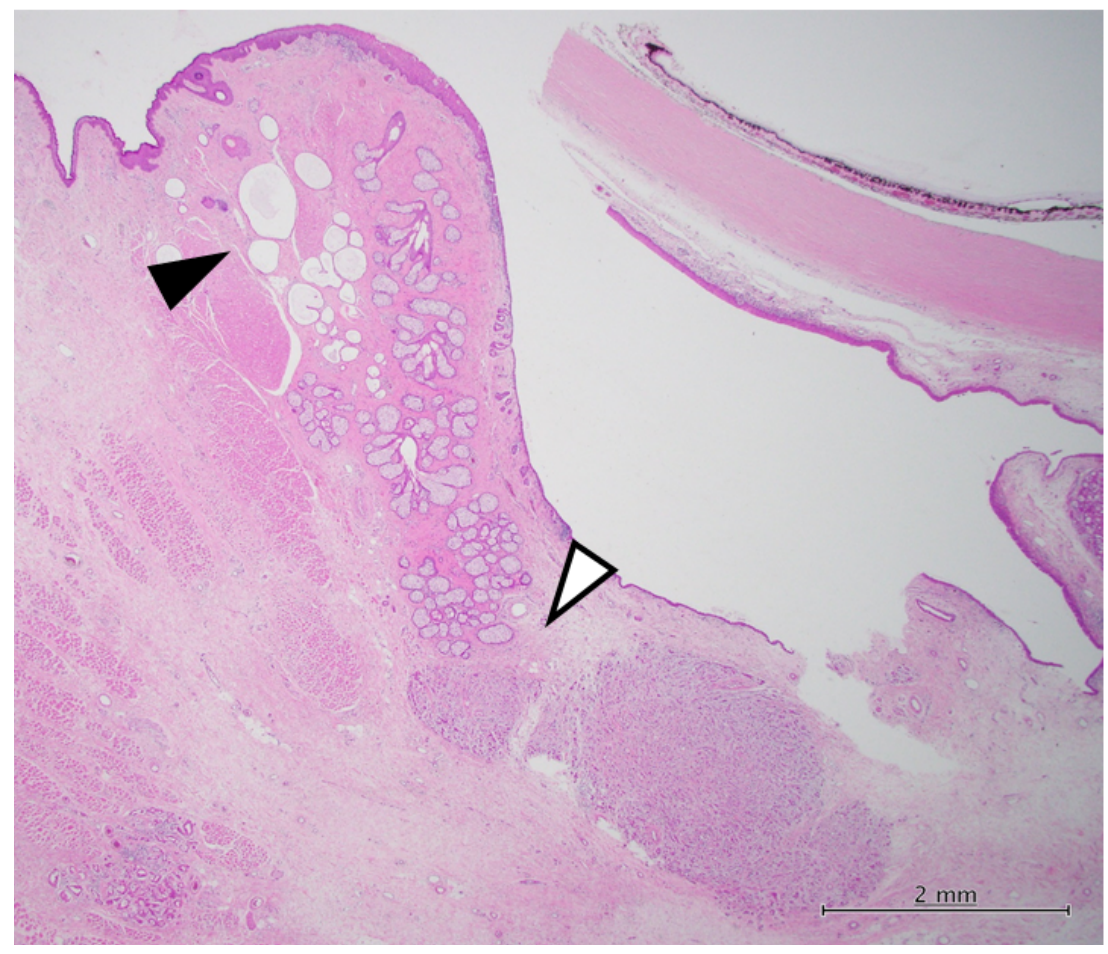

Figure 3: Microscopically, there are no tumor cells invading Moll's glands of the inferior eyelid. The black arrow indicates Moll's gland. The white arrow indicates lacrimal gland adenocarcinoma. 

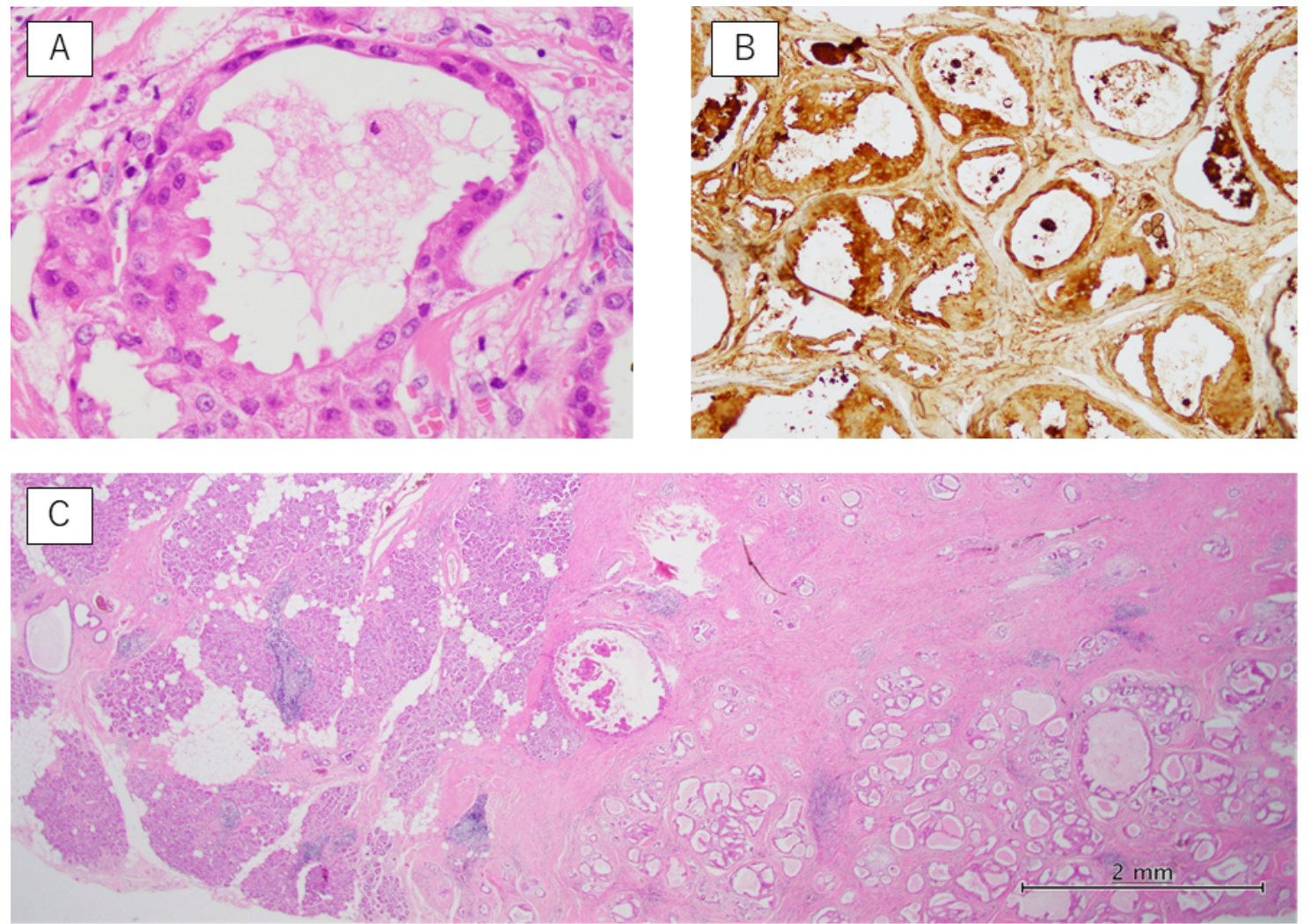

Figure 4: (a) Decapitation secretion; (b) Immunohistochemical staining showing GCDFP-15-positive cells. (c) Lacrimal gland adenocarcinoma with apocrine metaplasia. To the left is lacrimal gland adenocarcinoma. To the right is apocrine metaplasia.

rimal gland had acidophilic granular cytoplasm, decapitation secretion, and GCDFP-15 immunoreactivity. The zones with apocrine carcinoma characteristics within the adenocarcinomas lesion suggested that the tumor might have partially undergone apocrine metaplastic change. However, apocrine carcinoma's origin could not be determined; whether the cells with apocrine metaplasia became malignant or the tumor cells underwent apocrine metaplasia was not clear. There are no reports of apocrine carcinoma originating from the lacrimal gland; however, Lozano, et al. reported on a patient with lacrimal gland carcinoma with apocrine metaplasia [7]. Therefore, the patient was diagnosed with adenocarcinoma with apocrine metaplasia because the tumor cells did not invade Moll's glands, and apocrine metaplasia occupied less than $90 \%$ of the tumor.

In the view of prognosis, average survival of lacrimal gland carcinoma were reported one and a half years as local recurrence or distant metastasis arouse within a few years $[8,9]$. Multimodal therapy is administered to patients with localized disease, whereas wide surgical resection of the primary tumor and adjuvant therapy are considered for primary treatment. Regarding lymph node metastasis of lacrimal carcinoma, Jeong, et al. reported eyelid tumors or palpebral conjunctiva often metastasize to lymph nodes on the parotid gland surface; those in the lacrimal gland or lacrimal sac prefer the parotid gland and the anterior part of the auricle [10]. Hayashi, et al. reported that heavy particle beam treatment for lacrimal gland carcinoma achieved favorable outcomes, with five-year local control and overall survival rates of $62 \%$ and $65 \%$, respectively [11]. This approach, while achieving good local control and possible preservation of the eye, was associated with high rates of complications, including dysopia, cataract, and glaucoma. In the current case, radical resection was the primary treatment, due to its feasibility, and led to a good clinical course for three years after treatment. Based on a recent study reporting HER2 overexpression in malignant lacrimal carcinoma [12], novel treatment strategies with molecular targeted drugs should be considered in the future.

Due to the rarity of lacrimal gland adenocarcinoma, obtaining a sufficient sample size to evaluate evidence-based treatment strategies remains challenging. Future studies focusing on new treatment approaches are therefore warranted.

\section{Conclusion}

We herein presented a unique case of lacrimal gland adenocarcinoma in the inferior eyelid. Future investigation is necessary to further characterize lacrimal gland adenocarcinoma, a rare disease without established treatment strategies.

\section{Ethical Statement}

The authors declare that this study was conducted in accordance with the tenets of Declaration of Helsinki.

\section{Acknowledgments}

We thank Prof. Kouji Arihiro and Dr. Noriyuki Shiroma at the Department of Pathology in Hiroshima University for their guidance in pathological evaluation.

\section{Declaration of Interest Statement}

All authors declare that they have no conflicts of interest in relation to this study.

\section{References}

1. Shields JA, Shields CL, Scartozzi R (2004) Survey of 1264 Patients with Orbital Tumors and Simulating Lesions. Ophthalmology 111: 997-1008. 
2. Ohtsuka K, Hashimoto M, Suzuki Y (2005) A Review of 244 Orbital Tumors in Japanese Patients During a 21-Year Period: Origins and Locations. Jpn J Ophthalmol 49: 49-55.

3. von Holstein SL, Coupland SE, Briscoe D, Le Tourneau C, Heegaard S (2013) Epithelial tumours of the lacrimal gland: a clinical, histopathological, surgical and oncological survey. Acta Ophthalmol 91: 195-206.

4. Paties C, Taccagni GL, Papotti M, et al. (1993) Apocrine carcinoma of the skin. A clinicopathologic, immunocytochemical, and ultrastructural study. Cancer 71: 375-381.

5. Dhawan SS, Nanda VS, Grekin S, et al. (1990) Apocrine adenocarcinoma: Case report and review of the literature. J Dermatol Surg Oncol 16: 468-470.

6. Requena L, Kiryu H, Ackerman AB (1998) Neoplasms with apocrine differentiation, Philadelphia. Lippincott-Raven: 29-40.
7. Lozano M, Gonzalez F (2007) Adenocarcinoma with apocrine differentiation of the lacrimal grand. Arch Soc Esp Oftalmol 82: 229-232.

8. Touil A, El Abbassi S, Echchikhi Y, et al. (2017) Adenocarcinoma of the lacrimal gland: a case report. J Med Case Rep 11: 257.

9. Weis E, Rootman J, Joly TJ, et al. (2009) Epithelial lacrimal grand tumors pathologic classification and current understanding. Arch Ophthalmol 127: 1016-1028.

10. Jeong HS, Son YI, Baek CH (2006) The pattern of lymphatic metastasis of malignant tumors in the periorbital area. Am J Otolaryngol 27: 5-8.

11. Hayashi K, Koto M, Ikawa $\mathrm{H}$, et al. (2018) Efficacy and safety of carbon-ion radiotherapy for lacrimal grand carcinomas with extraorbital extension: a retrospective cohort study. Oncotarget 27: 12932-12940.

12. White VA (2012) Update on lacrimal gland neoplasms: Molecular pathology of interest. Saudi J Ophthalmol 26: 133-135. 